\title{
Disseminating Findings From EPC Reports: Pilot Project of Three Products
}




\title{
Disseminating Findings From EPC Reports: Pilot Project of Three Products
}

\author{
Prepared for: \\ Agency for Healthcare Research and Quality \\ U.S. Department of Health and Human Services \\ 5600 Fishers Lane \\ Rockville, MD 20857 \\ www.ahrq.gov
}

Contract No. 290-2015-00006-I

Prepared by:

Johns Hopkins University Evidence-based Practice Center

Baltimore, MD

Investigators:

Karen A. Robinson, Ph.D.

AHRQ Publication No. 18(19)-EHC024-EF

October 2018 


\section{Key Messages}

- Purpose of project: To conduct a pilot study of three dissemination products for an evidence report targeting decision makers in a health system.

- Findings: Key messages, as key points or newsletter item, were seen as useful by one of four respondents. Other responses suggest that the dissemination products were generally not considered informative. The other product, an Evidence to Decision (EtD) framework does not seem generally feasible, or useful, as a dissemination product for EPC reports.

- Lessons Learned for EPC Program: The nature of the evidence report and decision used in this pilot study limits specific applicability to other EPC reports.

- Utility for Health Systems: It is unclear what role dissemination products from evidence reports may play for specific decision makers and specific decisions if information not requested. 
This report is based on research conducted by the Johns Hopkins University EPC under contract to the Agency for Healthcare Research and Quality (AHRQ), Rockville, MD (Contract No. 2902015-00006-I). The findings and conclusions in this document are those of the authors, who are responsible for its contents; the findings and conclusions do not necessarily represent the views of AHRQ. Therefore, no statement in this report should be construed as an official position of AHRQ or of the U.S. Department of Health and Human Services.

\section{None of the investigators have any affiliations or financial involvement that conflicts with the material presented in this report.}

The information in this report is intended to help health care decisionmakers-patients and clinicians and health system leaders, among others-make well-informed decisions and thereby improve the quality of health care services. This report is not intended to be a substitute for the application of clinical judgment. Anyone who makes decisions concerning the provision of clinical care should consider this report in the same way as any medical reference and in conjunction with all other pertinent information (i.e., in the context of available resources and circumstances presented by individual patients).

This report is made available to the public under the terms of a licensing agreement between the author and the Agency for Healthcare Research and Quality. This report may be used and reprinted without permission except those copyrighted materials that are clearly noted in the report. Further reproduction of those copyrighted materials is prohibited without the express permission of copyright holders.

AHRQ or U.S. Department of Health and Human Services endorsement of any derivative products that may be developed from this report, such as clinical practice guidelines, other quality-enhancement tools, or reimbursement or coverage policies may not be stated or implied.

This report may periodically be assessed for the currency of conclusions. If an assessment is done, the resulting surveillance report describing the methodology and findings will be found on the Effective Health Care Program Web site at: www.effectivehealthcare.ahrq.gov. Search on the title of the report.

Persons using assistive technology may not be able to fully access information in this report. For assistance contact EffectiveHealthCare@ahrq.hhs.gov.

Suggested citation: Robinson KA. Disseminating Findings from EPC Reports: Pilot Project of Three Products. Methods Research Report. (Prepared by the Johns Hopkins University Evidencebased Practice Center under Contract No.290-2015-00006-I) AHRQ Publication No. 18(19)EHC024-EF. Rockville, MD: Agency for Healthcare Research and Quality; October 2018. Posted final reports are located on the Effective Health Care Program search page. DOI: https://doi.org/10.23970/AHRQEPCMETHENGAGEDISSEM. 


\section{Preface}

The Agency for Healthcare Research and Quality (AHRQ), through its Evidence-based Practice Centers (EPCs), sponsors the development of evidence reports and technology assessments to assist public- and private-sector organizations in their efforts to improve the quality of health care in the United States. The reports and assessments provide organizations with comprehensive, science-based information on common, costly medical conditions and new health care technologies and strategies. The EPCs systematically review the relevant scientific literature on topics assigned to them by AHRQ and conduct additional analyses when appropriate prior to developing their reports and assessments.

To improve the scientific rigor of these evidence reports, AHRQ supports empiric research by the EPCs to help understand or improve complex methodologic issues in systematic reviews. These methods research projects are intended to contribute to the research base in and be used to improve the science of systematic reviews. They are not intended to be guidance to the EPC program, although may be considered by EPCs along with other scientific research when determining EPC program methods guidance.

AHRQ expects that the EPC evidence reports and technology assessments will inform individual health plans, providers, and purchasers as well as the health care system as a whole by providing important information to help improve health care quality. The reports undergo peer review prior to their release as a final report.

If you have comments on this Methods Research Project they may be sent by mail to the Task Order Officer named below at: Agency for Healthcare Research and Quality, 5600 Fishers Lane, Rockville, MD 20857, or by email to epc@ahrq.hhs.gov.

Gopal Khanna, M.B.A.

Director

Agency for Healthcare Research and Quality
Arlene Bierman, M.D., M.S.

Director

Center for Evidence and Practice Improvement

Agency for Healthcare Research and Quality

Laura L. Pincock, Pharm.D., M.P.H.

Task Order Officer

Center for Evidence and Practice

Improvement

Agency for Healthcare Research and Quality 


\section{Disseminating Findings from EPC Reports: Pilot Project of Three Products}

\section{Structured Abstract}

Objectives. To conduct a pilot study of three dissemination products for an evidence report targeting decision makers in a health system.

Methods. We selected a recent evidence report that presented information about a specific decision. We then prepared three dissemination products for the report: key points, newsletter item, and an evidence to decision (EtD) framework. We identified and sought feedback from those in a health system who make the specific decision.

Results. We received responses from four of the five decision makers. The key points and newsletter item products were considered useful by one respondent. The other responses were not comprehensive but suggested that none of the products were helpful.

Conclusions. Dissemination alone or "pushing” information not requested does not seem to be seen as informative or useful by decision makers. Identifying what information to provide when, especially when not requested by the health system decision makers, needs further consideration. 


\section{Contents}

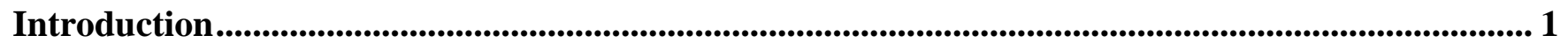

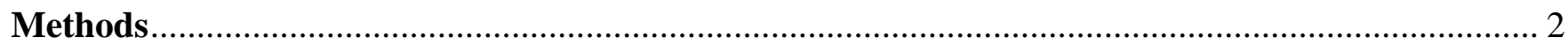

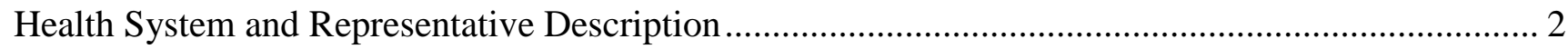

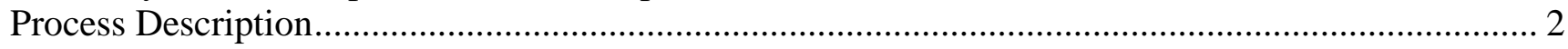

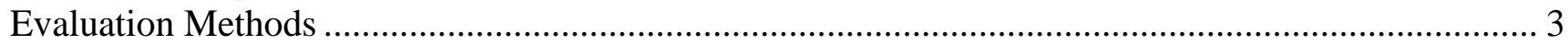

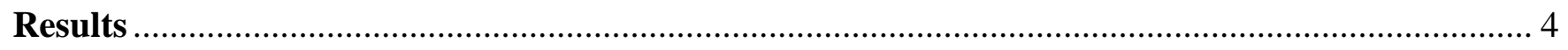

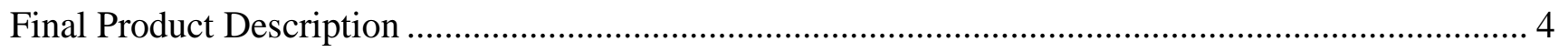

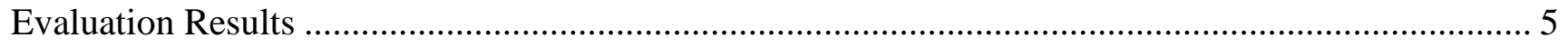

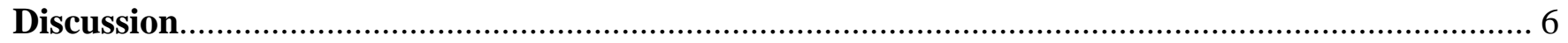

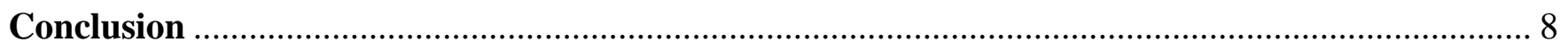

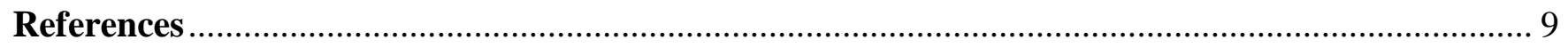

\section{Appendixes}

Appendix A. Dissemination Products 


\section{Introduction}

Since 1997, evidence reports produced by AHRQ Evidence-based Practice Centers (EPCs) have been used by various groups, such as professional organizations, and Federal agencies, to inform clinical practice guidelines, program planning, and research priorities. The AHRQ EPC program wants to improve the utility, use and uptake of existing EPC reports by health systems. Specifically, the program seeks to improve the uptake of existing EPC reports by health systems. To support this objective, EPCs conducted pilot methods projects to disseminate findings from existing EPC reports with an overall goal of helping health systems use EPC reports. The results from the pilot projects will also aid in refining and developing new or existing products, and in providing guidance for future reports and their associated products.For our pilot project, the Johns Hopkins University (JHU) EPC selected our prior evidence report "Contrast-Induced Nephropathy: Comparative Effects of Different Contrast Media”. ${ }^{1}$ In this report, we evaluated the comparative effects of different types of contrast media with respect to the risk of developing contrast-induced nephropathy (CIN). We chose to use this report as it was relatively current, and the choice of contrast type is a specific health system decision to target.We engaged with decision makers in the JHU health system to develop and evaluate three types of dissemination products based on the selected EPC report. 


\section{Methods}

\section{Health System and Representative Description}

We first sought guidance from the lead author of the selected report (our report that compared contrast media types). The lead author provided preliminary information about where the decisions are made to stock and recommend contrast media products within the JHU health system. We then used a modified snowball technique to identify additional individuals who make decisions on contrast media within the JHU health system.

In our institution, the decision about contrast media is made at the department level and thus this was the target for our work. Specifically, we sought to engage the managers and directors (i.e., the decision makers) of the departments that conduct computed tomography (CT) scans and magnetic resonance imaging (MRI).

We invited the individuals with the following roles to participate:
1. Clinical Director CT
2. Clinical Director MRI
3. Clinical Director Neuroradiology
4. Department Manager CT
5. Department Manager MRI

\section{Process Description}

Three dissemination products were prepared that presented the information from the evidence report in different formats:

- $\quad$ Key Points. We developed key points from the report following current EPC Program guidance. (The report on contrast media pre-dated the implementation of key points in evidence reports.)

- $\quad$ Newsletter item. We created a summary following the recommendation from the report of FY17 Workgroup 2 "Understanding Health-Systems Use of and Need for Evidence to Inform Decisionmaking”. ${ }^{2}$ Specifically, we created a brief newsletter entry that followed recommendation 1 from report: "Modify the dissemination emails that go out to health-systems to include not only the titles of the reports and hyperlinks to the full reports, but to include key messages (or hyperlinks to key messages) so that potential users can better assess the relevance of their report to their decision making and better triage the findings internally within their system”.

- Evidence to Decision (EtD) frameworks. We completed an EtD framework. EtD frameworks were developed for making clinical recommendations, coverage decisions, and health system or public health recommendations and decisions. This brief format is designed to provide information about the result, and strength of evidence, as well as additional contextual information such as cost, and patient values/preferences. ${ }^{3}$ 


\section{Evaluation Methods}

We contacted each decision maker via email. In the email, we presented each decision maker with the three products. The products were not labeled by type of product (i.e., file was not named "Key Points”) and were presented in random order. We asked a series of brief questions (see Box 1).

\section{Box 1. Questions}

Three products are attached: labeled A, B, and C.

Please provide feedback by responding to the following questions:

1. Was the information provided clear? Was one product easier to read?

2. Does the product provide enough information to make a decision or take action:
a. None of them did
b. All of them did
c. A did
d. B did
e. C did

3. What other types of information would have been helpful?

4. Are you likely to use one of these or a similar product to help make a decision?

5. Was one product more likely to prompt you to use the hyperlink to review the report?

6. Any other comments?

We made initial contact with the three directors January 19, 2018, to provide information about the project and to seek participation. The first email with the products was sent to decision makers May 30, 2018. Repeated reminders, followup emails, and calls were made until July 23, 2018. 


\section{Results}

\section{Final Product Description}

We reviewed the final evidence report to develop the products, focusing on the executive summary. We also reviewed the manuscript derived from the report. ${ }^{4}$ Draft versions of the products were reviewed by the lead author of the evidence report and by our Task Order Officer. Revisions were made based on those reviews and were primarily made to ensure accuracy of messages and consistency of content across the products.

The final products (key points, newsletter item, EtD) are included in the appendix. The key points and newsletter item products were fairly straightforward to develop. Because this report did not have existing key points we primarily spent time getting familiar with the report to determine what should be presented in the key points created specifically for this project. This took several hours for each product and relied on someone with training in systematic review methods, experience in drafting key messages and general understanding of the question being addressed in the report. As noted, guidance was requested from the lead author of the report to confirm accuracy and completeness of the materials drafted.

Completion of the EtD framework also relied on time to get familiar with the report content but posed additional challenges. There are several templates for the EtD framework, each with different components based on the decision being informed. We selected the template for coverage decisions as this was the closest fit to the decision our report could inform. ${ }^{5}$ The EtD framework for coverage decisions is very similar to the framework for clinical decisions but structured for decisions at the population level. We felt that this was a better fit than the clinical decision framework since the decision makers were making decisions about contrast media for their department, and were not making decisions about specific patients.

The first part of the EtD framework outlines the background and the Population, Intervention, Comparison and Outcome (PICO) elements of the question or decision. The second part includes items specific to the context and setting of the decision. Our plan had been to complete all of these items, however, it became apparent that many items about context and setting need to be completed by the specific decision maker for the specific decision. The selected report also did not provide information about these items. This meant that several sections did not have content from the report. In these cases we inserted the question used to assess the item, with the thought that the questions could serve to prompt the decision maker to think through elements needed to make a decision (See Appendix). For instance, for 'Resource Use' we inserted that the decision maker was to determine this item and included "Need to consider costs of different media, as well as hospitalization costs, and costs to treat any adverse drug reactions." Similar text was used for the items of equity ("Any differences in effect or impact of one type of media or another expected for disadvantaged people?"), acceptability (“Is one type of media more acceptable to patients or providers?”) and feasibility ("Is one type of media more feasible to use?”).

Other sections of the EtD were not applicable due to the nature of the question addressed in the selected evidence report. For instance, the selected report did not assess the potential beneficial effects of the different types of media. Thus, for the criterion of "Benefits and harms" we inserted the relevant question ("how substantial are the expected effects?”) and noted that benefits were not assessed. 


\section{Evaluation Results}

Obtaining responses was extremely difficult. We sent multiple emails and made several phone calls over 3 months. We received 4 responses from 5 individuals contacted. Two respondents noted that the specific type of contrast media noted in the dissemination products are not used in their department. They agreed to review the products and respond to the questions considering if the specific product mentioned was relevant, however, multiple reminders were not successful in obtaining responses to questions. The responses from decision makers that we did receive were limited (see Box 2).

\section{Box 2. Responses received}

Respondent 1: "I have nothing to add. Nothing in [the products] adds to what everyone already know. I found [the products] to be of little value." When prompted for additional feedback: "Most of what you said is on the acr website or on www.ctisus.com or in the apps store"

Respondent 2:

1. Information clear: Yes. [Key Points] is the easiest to read, [EtD] is the least.

2. Does product provide enough information to make a decision or take action: newsletter

3. Other types of information: "Specific recommendations, if there are any"

4. Likely to use one or these or similar to make a decision: yes

5. Was one product more likely to prompt you to use hyperlink to review report: no

Respondent 3 and 4: Individuals noted that the specific type of contrast media in the dissemination products are not used in their department. No further response received. 


\section{Discussion}

We evaluated three dissemination products targeting front-line decision makers in a health system. We sought feedback on a current product, a proposed product (implemented by the EPC Program during conduct of this study) and a new product.

\section{Utility and Applicability for Other Health Systems}

The limited responses from the decision makers, while hindering our ability to evaluate the specific products, is itself a useful lesson. The lack of response to our specific questions suggests that the products were not seen as informative. We suggest two implications of these results as it pertains to the utility of these products for decision makers in health systems. First, the "pushing" of information that has not been requested may be problematic. If a decision maker has not expressed a need for evidence to make a decision, it is not clear what role any dissemination product for EPC reports could play. This also leads to the second point: identifying the decision makers in health systems may not be sufficient to impact decision making. The content and format of the information provided may not be as important as at which point in the decision making process it occurs and how the information is provided to decision makers.

\section{Lessons Learned and Applicability for Other EPC Reports}

The strength of this project - targeting specific individuals making a very specific decision - may limit the applicability for other EPC reports. The report we selected, and its findings, were different from most EPC reports. For instance, the selected report did not consider the balance of benefits and harms for a particular therapy. Instead, this was a report on a diagnostic issue, specifically about the potential for harm from the type of contrast media used in diagnosis. In addition to being very different from most evidence reports, this meant that the report and findings did not fit well in the EtD framework for decisions. Finally, the results of the evidence report were not surprising. There was no additional or different information provided that would change practice or cause someone to make a different decision.

Completing the products, especially the EtD, requires specific knowledge of the decision arena. The content of the framework is setting specific meaning that we were unable to complete the context specific items in the framework. It is not clear from our results if completing those items would make the decision makers in a health system consider the EtD useful. It may be worthwhile testing the usefulness of an EtD framework within a different process. For instance, at the end of a project, an EPC could engage the partner/decision maker in completing the framework. This would enable the completion of the framework with specific decision contextual information. The act of completing the framework together may also, in itself, promote greater utility of the report and, ultimately, promote its dissemination and implementation.

Based on the responses received, and on work of prior workgroups, decision makers in health systems want to be provided with what to do - they want a simple answer. For instance, one respondent noted using an app to inform decisions and another respondent liked the key messages but when asked about what other information they would like to see in the products said that they wanted recommendations. This may suggest a need to manage expectations about what the dissemination products can be or can provide. It also suggests that a challenge for the EPC program will be to respond to the desire of decision makers to have bottom-line statements about "what to do" within the structure a systematic review. We see a few potential obstacles in trying to meet this challenge. First, it is difficult even with the space available in the report or manuscripts to present the nuances in the certainty of 
conclusions or strength of evidence. To do so in a summary dissemination product, especially when the user wants a bottom-line statement, may be impossible for some reports or for some questions within reports. Second, there is a risk that succinct bottom-line statements may be interpreted as a recommendation. This would be misleading and could put the EPC Program in an awkward or vulnerable position. 


\section{Conclusion}

Our findings provide some support for current approaches to dissemination, including the utility of the key messages, as key points or newsletter products. It does not seem feasible that the EtD framework be used for other EPC reports except in very specific circumstances in collaboration with the user of the report.

Dissemination alone or "pushing" information not requested does not seem to be seen as informative or useful by decision makers. It is not clear what role a dissemination product of an EPC report may play if the decision maker is not seeking information. Identifying what information to provide when, especially when not requested by the health system decision makers, needs further consideration. 


\section{References}

1. Eng J, Subramaniam RM, Wilson RF, et al. Contrast-Induced Nephropathy: Comparative Effects of Different Contrast Media. Comparative Effectiveness Review No. 155. (Prepared by the Johns Hopkins University Evidence-based Practice Center under Contract No. 290-201200007-I.) AHRQ Publication No. 15(16)EHC022-EF. Rockville, MD: Agency for Healthcare Research and Quality; December 2015. PMID: 26854293

2. White CM, Sanders Schmidler GD, Butler M, et al. Understanding Health-Systems' Use of and Need for Evidence to Inform Decisionmaking. Research White Paper. (Prepared by the University of Connecticut and Duke Evidencebased Practice Centers under Contract No. 2902015-00012-I and 290-2015-00004-I.) AHRQ Publication No. 17(18)-EHC035-EF. Rockville, MD: Agency for Healthcare Research and Quality; October 2017. PMID: 29611913.
3. Alonso-Coello P, Schunemann HJ, Moberg J, et al. GRADE Evidence to Decision (EtD) frameworks: a systematic and transparent approach to making well informed healthcare choices. 1: Introduction. BMJ. 2016 Jun 28;353:i2016. PMID: 27353417. doi: 10.1136/bmj.i2016.

4. Eng J, Wilson RF, Subramaniam RM, et al. Comparative Effect of Contrast Media Type on the Incidence of Contrast-Induced Nephropathy: A Systematic Review and Meta-analysis. Ann Intern Med. 2016 Mar 15;164(6):417-24. PMID: 26830055. doi: 10.7326/M15-1402

5. Parmelli E, Amato L, Oxman AD, et al. Grade Evidence to Decision (EtD) Framework for Coverage Decisions. Int J Technol Assess Health Care. 2017 Jan;33(2):176-82. PMID: 28655365. doi: $10.1017 / S 0266462317000447$ 


\section{Appendix A. Dissemination Products}

Key Points

Newsletter item

Evidence to Decision (EtD) Framework

From: Agency for Healthcare Research and Quality

\section{$\underline{\text { Key Points }}$}

\section{Purpose of review:}

To compare contrast-induced nephropathy (CIN) risk for contrast media within and between osmolality classes in patients receiving diagnostic or therapeutic imaging procedures.

\section{Key messages:}

- Iodixanol (iso-molar contrast media (IOCM)) had a slightly lower risk for CIN than a diverse group of low-osmolar contrast media (LOCM), but the lower risk did not exceed a minimally important clinical difference (moderate strength of evidence (SOE)).

- There was no difference in risk of CIN between different types of LOCM, but there is low confidence in the evidence (low SOE).

- There was no relationship between the route of administration and comparative CIN risk.

- There was no difference between IOCM and LOCM for secondary outcomes, such as cardiac events, need for renal replacement therapy, and mortality.

For more, see: https://effectivehealthcare.ahrq.gov/topics/nephropathy-contrastinduced/research/ 
From: Agency for Healthcare Research and Quality

\section{Evidence Review Now Available}

Contrast-Induced Nephropathy: Comparative Effects of Different Contrast Media (systematic review, released December 2015)

\section{Selected key findings:}

- There was a slightly lower risk for CIN with iso-osmolar contrast media (IOCM) (i.e., iodixnol) compared with low-osmolar contrast media (LOCM).

- No difference in risk of CIN was found between different LOCM.

- No relationship was found between routes of administration (intra-arterial versus intravenous) and CIN risk.

- Contrast dose or patient characteristics did not affect the risk of CIN.

- For more, see: https://effectivehealthcare.ahrq.gov/topics/nephropathy-contrastinduced/research/ 
From: Agency for Healthcare Research and Quality

\section{Evidence to Decision Framework (EtD): Question Section}

Question: Does different contrast media, within and between osmality classes, affect the risk of contrast-induced nephropathy (CIN) in patients receiving diagnostic or therapeutic imaging procedures?

Population: all patients (children and adults) undergoing procedures or diagnostic studies requiring administration of contrast media.

Intervention: contrast media

\section{Comparison:}

- $\quad$ low-osmolar contrast media (LOCM) vs. LOCM

- $\quad$ LOCM vs. iso-osmolar contrast media (IOCM)

\section{Main Outcomes:}

- $\quad$ contrast-induced nephropathy (CIN)

- Secondary outcomes: patient characteristics (renal replacement therapy, cardiac outcomes, adverse events, and mortality)

Setting: Inpatient and outpatient populations

Perspective: health system

Subgroups: patients with high-risk for nephropathy (based on age, cardiovascular and other comorbidities, creatinine levels) versus low-risk patients

\section{Background:}

- $\quad$ Radiological studies using iodine-contrast are an important and common clinical diagnostic tool but best ways to avoid the risk of contrast-induced nephropathy is a clinical challenge duet to the lack of clear scientific evidence.

For more, see AHRQ Evidence Review:

https://effectivehealthcare.ahrq.gov/topics/nephropathy-contrast-induced/research/ 
Criteria for EtD: Coverage Decisions

\begin{tabular}{|c|c|}
\hline Criterion & \\
\hline Priority of problem & There is recent doubt as to existence and significance of CIN. \\
\hline Benefits and harms & $\begin{array}{l}\text { How substantial are the expected effects? (benefits not } \\
\text { assessed) }\end{array}$ \\
\hline Certainty of evidence & $\begin{array}{l}\text { Iodixanol (iso-molar contrast media (IOCM)) had a slightly } \\
\text { lower risk for CIN than a diverse group of low-osmolar } \\
\text { contrast media (LOCM), but the lower risk did not exceed a } \\
\text { minimally important clinical difference (moderate strength of } \\
\text { evidence (SOE)). } \\
\text { There was no difference in risk of CIN between LOCM but } \\
\text { there is low confidence in the evidence (low SOE). }\end{array}$ \\
\hline Outcome importance & $\begin{array}{l}\text { Decision-maker to determine: How much do people value } \\
\text { avoiding CIN? }\end{array}$ \\
\hline Balance & $\begin{array}{l}\text { Decision-maker to determine: Does balance of desirable and } \\
\text { undesirable effects favor one type of media? (benefit not } \\
\text { assessed) }\end{array}$ \\
\hline Resource use & $\begin{array}{l}\text { Decision-maker to determine: Need to consider costs of } \\
\text { different media, as well as hospitalization costs, and costs to } \\
\text { treat any adverse drug reactions. }\end{array}$ \\
\hline Equity & $\begin{array}{l}\text { Decision-maker to determine: Any differences in effect or } \\
\text { impact of one type of media or another expected for } \\
\text { disadvantaged people? }\end{array}$ \\
\hline Acceptability & $\begin{array}{l}\text { Decision-maker to determine: Is one type of media more } \\
\text { acceptable to patients or providers? }\end{array}$ \\
\hline Feasibility & $\begin{array}{l}\text { Decision-maker to determine: Is one type of media more } \\
\text { feasible to use? }\end{array}$ \\
\hline
\end{tabular}

